\title{
CONF-9706113--12 \\ IMPLICATIONS OF ELECTRON ATTACHMENT TO HIGHLY-EXCITED STATES \\ IN PULSED-POWER DISCHARGES
}

\author{
Lal A. Pinnaduwage \\ Oak Ridge National Laboratory \\ P. O. Box 2008, Oak Ridge, Tennessee 37831-6122 \\ and \\ Department of Physics, University of Tennessee, Knoxville \\ Knoxville, Tennessee 37996

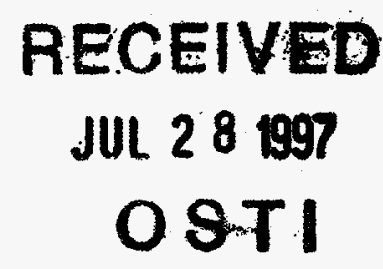

We point out the possible implications of electron attachment to highly-excited states of molecules in two pulsed power technologies. One involves the pulsed $\mathrm{H}_{2}$ discharges used for the generation of $\mathrm{H}^{\prime}$ ion beams for magnetic fusion energy and particle accelerators. The other is the power modulated plasma discharges used for material processing.

\section{INTRODUCTION}

\section{A. Background}

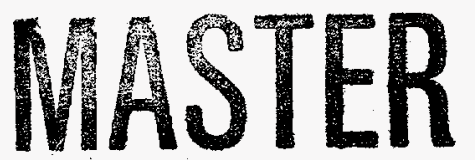

During the recent years evidence has emerged from two different research areas that employ direct current (DC) or radio frequency (RF) discharges, that improved performance for the respective processes can be obtained in time-modulated discharges compared to continuous discharges:

(i) in DC discharges used for $\mathrm{H}^{*}$ generation for fusion energy research, pulsed discharges yield higher time-averaged $\mathrm{H}^{-}$currents compared to continuous (cw) discharges $[1,2]$

(ii) in (mostly) RF discharges used for plasma processing of semiconductor material, timemodulated discharges yield faster deposition/etching and improved quality [3-7].

The mechanisms responsible for (i) and (ii) above have not been fully explained.

We present evidence that the enhanced performance in both of the above cases could be due to the enhanced dissociative electron attachment that occurs at the falling edge of a pulse in the case of a DC discharge (or at the turning off of the RF discharge). While the category (i) above directly depends on the enhanced electron attachment, in the following we show that the effects in the category (ii) are also indirectly related to the enhanced electron attachment.

It is only recently that the presence of significant populations of negative ions in plasma processing discharges was established, see the references in [8]. Since negative ions (especially radical anions) are very reactive in nature, one might expect them to participate actively in reactions that occur in processing discharges, and there is scattered evidence that in some cases negative ions can etch or deposit material better than positive ions or neutrals, see the references in [8]. At present it is generally believed that neutral radicals are mainly responsible for etching and deposition; in this context too, an enhanced dissociative electron attachment (DEA) process can play a significant role since it leads to radical production in addition to negative ion formation. Therefore, it is logical to state that enhanced DEA processes will be beneficial for etching/deposition.

Large negative ion densities in plasma processing discharges can also lead to an adverse effect, i.e., particulate formation. Even though modern clean rooms have reduced the once appreciable contamination caused by ambient exposure, the particulate generation by processing discharges themselves remains a problem. There have been numerous studies to show that plasma generation of particulates is the 


\section{DISCLAMIER}

Portions of this docament may be illegible in electronic image products. Images are produced from the best available original document. 


\section{DISCLAIMER}

This report was prepared as an account of work sponsored by an agency of the United States Government. Neither the United States Government nor any agency thereof, nor any of their employees, make any warranty, express or implied, or assumes any legal liability or responsibility for the accuracy, completeness, or usefulness of any information, apparatus, product, or process disclosed, or represents that its use would not infringe privately owned rights. Reference herein to any specific commercial product, process, or service by trade name, trademark, manufacturer, or otherwise does not necessarily constitute or imply its endorsement, recommendation, or favoring by the United States Government or any agency thereof. The views and opinions of authors expressed herein do not necessarily state or reflect those of the United States Government or any agency thereof. 
major source of wafer contamination during device fabrication. There is increasing evidence that negative ions are precursors for particulate formation in processing discharges, see the references in [8].

In a cw discharge, the negatively-charged particles are trapped in the discharge due to the sheath potentials and are allowed to grow into particulates. Once they become mocrometer-size particulates, the viscous drag force becomes large enough to overcome the electrostatic forces and they can reach the substrate. Therefore, if the negative ions do contribute deposition/etching, the cw operation of the discharge is detrimental in two ways: (i) it prevents the negative ions from reaching the substrate and thus contributing to the etching/deposition, and (ii) it traps the negative ions in the discharge, thus allowing them to grow to $\mu \mathrm{m}$-size particulates. By time-modulating the discharge, the negative ions populated during the "on-timen are allowed to reach the substrate during the "off-time" and thus deposition/etching is enhanced while particulate growth is prevented. In Section III, we will also provide evidence that in addition to the above processes, the negative-ion formation itself may be enhanced at the time the discharge is turned off and thus the flux of negative ions to the substrate is enhanced during the discharge off period.

In the remainder of Section I, we point out the mechanisms by which highly-excited states of molecules can be populated in gas discharges, and summarize the recent findings on DEA to highly-excited molecules. An example of the implications of enhanced DEA will be presented at the end of Section I. Then, in Sections II and III, we will describe our proposed mechanism to explain the recent observations in pulsed $\mathrm{H}_{2}$ discharges and in time-modulated discharges used for plasma processing of materials. The conclusions will be summarized in Section IV.

\section{B. Excitation of Long-Lived Excited States Molecules in Gas Discharges}

\section{Excitation via Electron Impact}

In gas discharges, highly-excited states of molecules can be populated via direct electron impact. Platzman [9] has shown that most of the oscillator strength for a given molecule is associated with transitions to energies around the ionization threshold, including energies $1-2 \mathrm{eV}$ below threshold where congestion of the bound states is high. These states are high-Rydberg (HR) states that can have lifetimes up to microseconds [10]. For example, long-lived HR states of $\mathrm{H}_{2}$ were shown to be populated in $\mathrm{H}_{2}$ discharges, see for example, $[11,12]$. Therefore, it is quite likely that substantial number densities of fairly long-lived highly-excited states of molecules are populated in gas discharges. Recent studies show that these HR states have extremely large cross sections for electron attachment, see Section I.C. below.

Another category of long-lived states produced in a gas discharge is the metatstable states of atoms and molecules. Most of the available data on electronically-excited states is restricted to the longlived metastable states of the rare gases that can be efficiently generated in gas discharge; in a typical rare gas plasma, the relative density of metastable to ground-state atoms is about $10^{-4}$, while the degree of ionization with respect to the ground-state population is only about $10^{-5}$ [13]. The available data on the electron collisions with metastable states of rare gases [13-15] and molecular gases [13] have been summarized.

\section{Excitation of Molecules via Excitation Transfer from Rare-Gas Metastables}

In addition to having large cross sections for electron collisions, the metastable states of atoms/molecules possess large cross sections for interactions with polyatomic molecules. Ground-state rare gases are inert, but the metastable states (which have electronic configuration of alkali atoms) are highly reactive; they transfer their excess energy to a wide variety of molecules with cross sections of $10^{-16}$ to $10^{-14}$ $\mathrm{cm}^{2}$ leading to excitation/ionization/fragmentation of those molecules [16, 17]. One aspect of this excitation transfer process (sometimes referred to as the Penning excitation process) that we wish to highlight here is the consequences of the excitation of a large number of highly-excited molecules in gas discharges. When the ionization threshold of the recipient molecule is close to the energy of the metastable state of the donor atom/molecule, highly-excited states of the recipient molecule are populated via excitation transfer. 


\section{Enhanced Electron Attachment to Highly-Excited Molecules}

From the Sections A and B above, it is clear that significant populations of excited molecular states are likely to be populated in discharges of unitary gases or gas mixtures with rare gases.

Studies conducted during the past few years show that slow electrons are efficiently attached to molecules laser-excited to energies above and close to their ionization thresholds; see $[8,18]$ and references therein. It appears $[8,18]$ that the electron attaching states are highly-excited (Rydberg) states that are indirectly populated via internal conversion from the laser-excited superexcited states. This has recently been confirmed [19].

Evidence for electron attachment to highly-excited molecules in a gas discharges was recently pointed out by us [20] in the elegant experiments conducted by lizuka et al. [21]: In these experiments a flowing Ar plasma was produced using a modified hollow-cathode discharge where Ar plasma was fed into the discharge through a hole in the cathode. This plasma was extracted into a "target region" through a hole in the anode; $\mathrm{CH}_{4}$ was fed into this target region. It is important to note that $\mathrm{CH}_{4}$ was not subjected directly to the discharge. It was shown [21] that of the electrons that were present in the target region in the absence of $\mathrm{CH}_{4}, 70-80 \%$ were converted to $\mathrm{H}^{-}$anions when $\mathrm{CH}_{4}$ was added; $\mathrm{H}$ ion densities of the order of $10^{9} \mathrm{~cm}^{-3}$ were reported [21] in the target region where the electron temperature was $<0.5 \mathrm{eV}[21,22]$. We pointed out [20] that the following sequence of events can explain their observations: (i) the flowing plasma carried the long-lived metastable Ar atoms, $\mathrm{Ar}^{\mathrm{m}}$ (energies $\sim 11.7 \mathrm{eV}$ ), to the target region, (ii) highly excited $\mathrm{CH}_{4}{ }^{*}$ states of $\mathrm{CH}_{4}$ (ionization threshold $\sim 12.6 \mathrm{eV}$ were produced via excitation transfer from $\mathrm{Ar}^{\mathrm{m}}$ atoms, and (iii) low-energy electrons in the target region attached dissociatively to $\mathrm{CH}_{4}{ }^{-}$, i.e., $\mathrm{e}^{-}+\mathrm{CH}_{4}{ }_{4}^{-} \mathrm{H}^{-}+\mathrm{CH}_{3}$ consistent with our observations [23] of efficient $H$ - formation via attachment of low-energy electrons to laser-excited states of $\mathrm{CH}_{4}$; our proposed mechanism is also consistent with lizuka et al.'s observation [24] of efficient $\mathrm{CH}_{3}$ radical formation in the same discharge source.

\section{Pulsed vs. Continuous Discharges}

In Sections A and B above, we showed that highly-excited states of molecules can be populated during the discharge via either electron impact or via excitation transfer from the metatstable states of an additive gas. Yet these highly-excited states are also likely to undergo efficient ionization/dissociation with collisions with energetic electrons in the discharge.

However, immediately following the termination of a discharge, the energetic electrons in the discharge start losing energy via collisions with gas atoms/molecules, and also by diffusion. If the highlyexcited states produced just before the termination of the discharge have long lifetimes then they will be exposed to slow electrons in the post discharge, i.e., conditions for electron attachment will dominate in the post discharge. Furthermore, in a mixture of rare gas/ molecular gas, the highly-excited states of the molecular gas will continue to be populated via excitation transfer from the metastable states of the rare gas in the post discharge.

Some of the above discussed ideas have been used to explain experimental observations in an entirely different area as described below.

\section{E. Role of the Metastable States of Rare-Gases in Glow Discharge Mass Spectrometry}

Glow discharge mass spectrometry is predominantly used as an analytical technique for the detection of trace elements in solid materials [25]. In these experiments, the solid sample to be analyzed serves as the cathode, and a glow discharge takes place in in a rare-gas environment; collisions with electrons and metastable states of rare-gas atoms cause ionization of the sputtered atomic species, which are mass analyzed. Even prior to the studies on pulsed discharges, evidence for the dominant role played by the metastable states of the rare gases in the continuous operation of the above discharges had been presented [26, 27]: In the first study [26], depopulation of the metastable states (via laser irradiation) was shown to lower ion signals from species with ionization potentials less than the metastable energy; however, signals due to species with ionization potentials higher than the metastable energy were not affected. In the second study [27], it was shown that approximately $40 \%$ to $80 \%$ of the ionization of the sputtered species 
was due to collisions with the metastable states (Penning ionization).

The role of the metastable states was shown to be enhanced in the pulsed operation of the discharge $[28,29]$. This was shown to be due to two reasons: (i) upon discharge termination, electrons thermalize rapidly and thus the metastable rare-gas atoms no longer experience collisions with electrons sufficiently energetic to quench their population [29], (ii) the rapid recombination of rare-gas ions with thermalized electrons results in a production of metastable rare-gas atoms [30] in the post discharge. Therefore, the ionization of the sputtered atomic species that remained in the discharge region was enhanced in the post discharge.

\section{PULSED HYDROGEN DISCHARGES}

Generation of $\mathrm{H}^{-}$ions using $\mathrm{H}_{2}$ discharges is actively being pursued due to the need for intense $\mathrm{H}^{\circ}$ ion beams for particle accelerators and for magnetic fusion energy research. Enhanced electron attachment to vibrationally-excited states of the ground electronic state of $H_{2}$ was reported $[31,32]$ at about the same time the efficient $\mathrm{H}^{-}$formation in $\mathrm{H}_{2}$ discharges was reported [33]. Ever since that time, an exhaustive experimental and theoretical effort has been devoted to understand the experimental observations in the $\mathrm{H}_{2}$ discharge sources in terms of this electron attachment process (see the reviews in [34-36]). However, recent studies [37-39] show that the experimentally measured $\mathrm{H}$ number densities can not be completely accounted for by electron attachment to high-vibrational (HV) states. Based on our observations on electron attachment to laser-excited $\mathrm{H}_{2}$ [40], we recently pointed out [41] that highly-excited electronic states of $\mathrm{H}_{2}$, which are high-Rydberg (HR) states, populated in $\mathrm{H}_{2}$ discharges may account for the difference, also see [18]. Recent attempts [Garscadden, $1995 \# 130$; Hiskes, $1995 \# 131$ ] to evaluate the relative contributions of the HV and $\mathrm{HR}$ states for $\mathrm{H}^{-}$formation in $\mathrm{H}_{2}$ discharges have not led to a firm conclusion.

It has been recently shown $[1,2]$ that higher average $\mathrm{H}^{-}$beam currents can be extracted by timemodulating $\mathrm{H}_{2}$ discharge sources, i.e., by on-off modulation of the discharge voltage. Subsequent studies [42-44] have confirmed these observations and have presented further experimental observations designed to understand the reasons for this enhancement. Model calculations based on electron attachment to HV states yield $\mathrm{H}^{-}$ion yields that are at least 4 times smaller than the experimentally-measured values [45].

Another possibility to consider is the population of $\mathrm{HV}$ states via recombination of $\mathrm{H}_{3}{ }^{+}$ions with slow electrons in the post discharge (the $\mathrm{H}_{2}{ }^{+}$ions produced by ionization of $\mathrm{H}_{2}$ are quickly converted to $\mathrm{H}_{3}{ }^{+}$ions by fast ion-molecule reactions [41].) An upper bound for the recombination rate constants for the $\mathrm{H}_{3}{ }^{+}$ions is $\sim 2 \times 10^{-7} \mathrm{~cm}^{3} \mathrm{~s}^{1}$ [46] For a density of $\sim 10^{1} \mathrm{~cm}^{3}$ of $\mathrm{H}_{3}^{+}$ions corresponding to the Fig. 4 of [43], this yields a time of $z 50 \mu \mathrm{s}$ for the formation of HV states. However, the $\mathrm{H}^{-}$ion signal in Fig. 4 of [43] peaks at $\sim 10$ $20 \mu \mathrm{s}$. Therefore, the observed enhancement in $\mathrm{H}^{-}$signal cannot be due to the enhanced production of $\mathrm{HV}$ states in the post discharge.

We believe that the enhanced production of $\mathrm{H}^{-}$signal in [43], as well as in the other pulsed $\mathrm{H}_{2}$ discharge experiments is due to ability of the HR states to survive for longer times in the post discharge due to the quick removal (within $\sim 1 \mu \mathrm{s}$ ) of the energetic electrons from the discharge in the post discharge period.

\section{.III. TIME-MODULATED PLASMA PROCESSING DISCHARGES}

Most plasma processing discharges use gas mixtures consisting of a "reactive gas" and an inert gas. Thus in addition to the population of excited states of the reactive gas by electron impact, they can also be populated by excitation transfer from metastable states of the inert gas.

By monitoring the electron decay time in the afterglow of a silane/helium pulsed discharge, Fleddermann et al. [47] have shown that the dominant electron loss mechanism in the afterglow was dissociative electron attachment (non-dissociative electron attachment is not viable at the low pressures used in these experiments.) They estimated [47] a lower bound value of $\sim 2.6 \times 10^{-10} \mathrm{~cm}^{3} \mathrm{~s}^{-1}$ for the electron attachment rate constant involved; yet this is an order of magnitude higher than the electron attachment rate 
constant corresponding to the measured electron attachment cross sections measured for the ground state of silane [48]. Fleddermann et al.[47] proposed that this enhanced electron attachment in the afterglow was to $\mathrm{SiH}_{3}$ or $\mathrm{SiH}_{2}$ radicals that were produced during the discharge. However, this possibility can be ruled out as shown below.

Negative ions from a pulsed silane discharge have been mass identified recently by Howling et al. $[49,50]$. They reported that monosilicon negative ions are initially produced by electron attachment and subsequently these polymerize to produce polysilicon negative ions; the dominant monosilicon negative ion produced by electron attachment is $\mathrm{SiH}_{3}^{-}[49,50]$. Since it is not possible to produce the $\mathrm{SiH}_{3}^{-}$ion via dissociative electron attachment to $\mathrm{SiH}_{3}$ or $\mathrm{SiH}_{2}$ radicals, it is clear that the enhanced production of $\mathrm{SiH}_{3}{ }_{3}^{-}$ion in the afterglow must come from the parent $\mathrm{SiH}_{4}$ molecule. Since the ground state electron attachment for silane is more than an order of magnitude smaller than the experimentally measured value in the afterglow of a pulsed discharge [47], the only other explanation is dissociative electron attachment to highly-excited states of silane. This is also consistent with our recent observation [8] of large electron attachment rate constants of the order of $10^{-7} \mathrm{~cm}^{3} \mathrm{~s}^{-1}$ for highly-excited states of silane produced via laser irradiation.

\section{CONCLUSIONS}

We pointed out the possible significance of electron attachment to highly-excited states of molecules in two pulsed power technologies. One was in the pulsed discharges used for the generation of $\mathrm{H}^{-}$ion beams, and the other was in the time-modulated discharges used for the plasma processing of materials. We presented evidence from recent experiments which show that observed negative-ion formation in certain pulsed discharges can not be explained by electron attachment to molecules in their ground electronic states, and that the experimental observations are consistent with negative-ion formation via electron attachment to highly-excited molecules produced in the discharge-on periods. The existing basic electron attachment measurements on highly-excited molecules confirm that they have orders of magnitude enhancement in electron attachment compared to the respective ground states. The mechanisms by which highly-excited states of molecules may be populated in pulsed discharges were discussed.

This research is supported by the Environmental Management Science Program (EMSP) of the Department of Energy under a contract with the Oak Ridge National Laboratory, and by the National Science Foundation under contract ECS-9626217 with the University of Tennessee, Knoxville. The Oak Ridge National Laboratory is managed by Lockheed Martin Energy Research Corp. for the U. S. Department of Energy under contract number DE-AC05-96OR22464.

\section{REFERENCES}

1. M. B. Hopkins and K. N. Mellon, Phys. Rev. Lett. 67, 449 (1991).

2. M. B. Hopkins, M. Bacal and K. N. Mellon, J. Appl. Phys. 70, 2009 (1991).

3. R. W. Boswell and D. Henry, Appl. Phys. Lett. 47, 1095 (1985).

4. L. J. Overzet and J. T. Verdeyen, Appl. Phys. Lett. 48, 695 (1986).

5. S. Samukawa and T. Mieno, Plasma Sources Sci. Technol. 5, 132 (1996).

6. T. H. Ahn, K. Nakamura and H. Sugai, Plasma Sources Sci. Technol. 5, 139 (1996).

7. A. Hatta, H. Suzuki, K. Kadota, H. Makita, T. Ito and A. Hiraki, Plasma Sources Sci. Technol. 5, 235 (1996).

8. L. A. Pinnaduwage and P. G. Datskos, J. Appl. Phys. in press, (1997).

9. R. L. Platzman, Radiation Res. 17, 419 (1962).

10. R. F. Stebbings and F. B. Dunning, eds. Rydberg States of Atoms and Molecules, (Cambridge University Press, Cambridge, 1983).

11. S. M. Tarr, James A. Schiavone, and Robert S. Freund, J. Chem. Phys. 74, 2869 (1981).

12. U. Fano, J. Phys. B 7, L401 (1974). 
13. J. L. Delcroix, C. M. Ferreira and A. Ricard, in Principles of Laser Plasmas, G. Bakefi, Editor. 1976, Wiley and Sons: New York. p. 159.

14. C. C. Lin and L. W. Anderson, in Advances in Atomic, Molecular, and Optical Physics, D. Bates and B. Bederson, Editors. 1992, Academic Press, Inc.: New York. p. 1.

15. S. Trajmar and J. C. Nickel, in Advances in Atomic, Molecular, and Optical Physics, D. Bates and B. Bederson, Editors. 1993, Academic Press, Inc.: New York. p. 45.

16. L. G. Piper, J. E. Valazco and D. W. Sester, J. Chem. Phys. 59, 33231 (1973).

17. M. Bourene and J. L. Calve, J. Chem. Phys. 58, 1452 (1973).

18. P. G. Datskos, L. A. Pinnaduwage and J. F. Kielkopf, Phys. Rev. A 55, 4131 (1997).

19. L. A. Pinnaduwage and F. Zhu, Phys. Rev. Lett. (submitted, 1997).

20. L. A. Pinnaduwage, Appl. Phys. Lett. 67, 1034 (1995).

21. S. lizuka, T. Koizumi, T. Takada and N. Sato, Appl. Phys. Lett. 63, 1619 (1993).

22. N. Sato, S. lizuka, T. Koizumi and T. Takada, Appl. Phys. Lett. 62, 567 (1993).

23. L. A. Pinnaduwage, M. Z. Martin and L. G. Christophorou, Contrib. Plasma Phys. 35, 433 (1995).

24. S. lizuka, T. Takada and N. Sato, Appl. Phys. Lett. 64, 1786 (1994).

25. W. W. Harrison, K. R. Hess, R. K. Marcus and F. L. King, Ana. Chem. 58, 341A (1986).

26. K. R. Hess and W. W. Harrison, Ana. Chem. 60, 691 (1988).

27. R. L. Smith, D. Serxner and K. R. Hess, Ana. Chem. 61, 1103 (1989).

28. J. A. Klingler, P. J. Savickas and W. W. Harrison, J. Am. Soc. Mass Spectrom. 1, 138 (1990).

29. J. A. Klingler, C. M. Barshick and W. W. Harrison, Ana. Chem. 63, 2571 (1991).

30. F. L. King and C. Pan, Ana. Chem. 65, 735 (1993).

31. M. Allen and S. F. Wong, Physical Review Letters 41, 1791 (1978).

32. J. M. Wadehra and J. N. Bardsley, Phys. Rev. Lett. 41, 1795 (1978).

33. M. Bacal, and G. W. Hamilton, Phys. Rev. Lett. 42, 1538 (1979).

34. J. R. Hiskes, Comments At. Mol. Phys. 19, 59 (1987).

35. M. Bacal and D. A. Skinner, Comments At. Mol. Phys. 23, 283 (1990).

36. W. G. Graham, Plasma Sources Sci. Technol. 4, 281 (1995).

37. P. J. Eenshuistra, M. Gochitashvilli, R. Becker, A.W.Kleyn, and H.J.Hopman, J. Appl. Phys. 67, 85 (1990).

38. P. Berlemont, D. A. Skinner and M. Bacal, Rev. Sci. Instrum. 64, 2721 (1993).

39. A. T. Young, P. Chen, K. N. Leung and G. C. Stutzin, Rev. Sci. Instrum. 65, 1416 (1994).

40. L. A. Pinnaduwage and L. G. Christophorou, Phys. Rev. Lett. 70, 754 (1993).

41. L. A. Pinnaduwage and L. G. Christophorou, J. Appl. Phys. 76, 46 (1994).

42. R. M. A. Heeren, K. N. Mellon, M. B. Hopkins, D. Ciric and A. W. Kleyn, Europhys. Lett. 17, 503 (1992).

43. K. N. Mellon, B. P. Coonan and M. B. Hopkins, J. Phys. D 27, 2480 (1994).

44. K. N. Mellon, B. P. Coonan and M. B. Hopkins, Plasma Sources Sci. Technol. 4, 591 (1995).

45. M. B. Hopkins, . 1996.

46. T. Gougousi, R. Johnsen and M. F. Golde, Int. J. Mass Spectrom. Ion Phys. 149/150, 131 (1995).

47. C. B. Fleddermann, J. H. Beberman and J. T. Verdeyen, J. Appl. Phys. 58, 1344 (1985).

48. A. Garscadden, in Effects Due to Negative lons and particles in Plasmas, ed. by M. Capitelli and J. N. Bardsley (Plenum Press, New York, 1990), p. 541.

49. A. A. Howling, J.-L. Dorier and C. Hollenstein, Appl. Phys. Lett. 62, 1341 (1993).

50. A. A. Howling, L. Sansonnens, J.-L. Dorier and C. Hollenstein, J. Appl. Phys. 70, 2939 (1994). 